\title{
Editorial
}

\section{To identify the non-biological causes of child mortalities in developing countries, Social Autopsy tools should be based on "The Pathway to Survival Conceptual Framework". Muhammad Bilal Siddiqui ${ }^{1,2,3}$, Chiu Wan $\mathrm{Ng}^{4}$, Wah Yun Low ${ }^{5}$.}

\author{
${ }^{1}$ Head of "Centre of Maternal and Child Health Research" (CoMCHR) at Advanced Educational \\ Institute of Research Centre (AEIRC), \\ ${ }^{2}$ Director, Child Registry of Pakistan (CROP) \\ ${ }^{3}$ Senior Lecturer, Department of Community Health Sciences, Hamdard University, Karachi \\ Pakistan. \\ ${ }^{4}$ Department of Social and Preventive Medicine, University of Malaya, Kuala Lumpur, Malaysia. \\ ${ }^{5}$ Health Research Development Unit, Faculty of Medicine, University of Malaya. \\ Corresponding Author's Email: drbilals@gmail.com
}

\section{Keywords}

Child mortality, Social autopsy, Social determinants of Health, Conceptual framework, The Pathway to Survival Conceptual Framework.

Since a large proportion of child deaths occur due to preventable diseases in developing countries, estimating the cause of death (CoD) is very important for such prevention. Verbal Autopsy (VA) has given us tremendous amount of valuable data on the cause of death; however, VA data is primarily limited to assigning only biological cause of death (BCoD) to the unregistered child mortalities. Apart from gaining the information on $\mathrm{BCoD}$, knowing the social or non-biological determinants (NBDs) linked with health care seeking behavior, health care delivery and access to health care to any child is of utmost importance. This knowledge potentially may help us to develop interventions targeting effects of different barriers affecting the timely and appropriate health delivery to the needy, thereby may help us by armoring us with new and more advanced information to prevent the child mortalities and improving the child survival estimates in developing geographies, which have huge inconsistencies related to social determinants of health. The concept of capturing NBDs and relating them with the death incidents in children has been backed in the literature (1-10).

To record such NBD, there is a specific technique known as "Social Autopsy" (SA) (2, 6, 10-12), which as a tool, is similar to Verbal Autopsy (VA) tool; however, the only difference between the two is that SA tool focuses specifically on NBDs and VA focuses on assigning the $\mathrm{BCoD}$ in assigning the CoD of children who have died and due to any reason health/national database system was unable to get their CoD assigned.

Compared to VA, SA is comparatively a newer idea. SA focuses on capturing detailed information related to different NBDs using a structured questionnaire which are asked from parents or caretakers of deceased children (10). To capture and organize such determinants, the SA tool should be based on a well-constructed and relevant conceptual framework that should aid the proper data organization. To cater with, literature has shown several of the conceptual frameworks namely "The Three 
delay model", "SSSR", "The Pathway to Survival Conceptual Framework" etc.

In comparison to other CFs, literature has shown that "The Pathway to Survival Conceptual Framework" (TPtoSCF) provides a more holistic approach in capturing relevant information and later organizing it to give a much-detailed evidence and statistics on Non-biological causes of child mortalities (13). This framework was developed by joint efforts of Centers of Disease Control and Prevention (CDC) and United States Agency for International Development (USAID) in 1995, as a continuation of efforts for adding the social component in the maternal mortality investigations (13).

The Pathway to Survival Conceptual Framework (TPtoSCF) actually addresses all the relevant determinants discussed in different CFs, such as 'The Three Delay Model' (which only focuses on delays and related barriers during the case management at three interphases of maternal illness) and 'SSSR' (which is specifically related to undertaking SA on maternal deaths from a human right perspective); however, TPtoSCF organizes much more wider range of determinants and is specifically developed for organizing data on child mortalities.

TPtoSCF addresses following set of determinants which are worth to discuss (13).

1. Determinants related to preventive measure (towards child and mother) adopted by parents before the birth and illness (including breats feeding, immunization, tetanus vaccination of mothers before or during pregnancy etc.).

2. Case management at home; parents'/caretakers' health seeking behavior at home (including their knowledge; awareness and perception towards danger signs of common and fatal childhood ailments and behavior towards treatment adherence at home etc.).

3. Health care system and facility based determinants on case-management (including availability of services, and competence of health service providers).

4. Financial and socio-economic variables.

5. Transport based variables etc.

One of the most prominent characteristics of this framework is that it may be used for addressing the prevalence of different NBDs and their related barriers. This Framework has been built in keeping view of case management guidelines based on the Integrated Management of Childhood Illness (IMCI), making it most suitable for addressing the determinants related to child mortalities.

\section{Conclusion}

To wrap up:

1. The social causes of death are very important to gather.

2. The captured data should address as many social determinants as possible.

3. Social autopsy is a tool that specifically developed to gather different NBDs of mortalities.

4. Social autopsy should be based on a holistic conceptual framework relevant to the topic of interest. In our case, the most suitable CF relevant to the issue of identifying social causes of child mortality is The Pathway to Survival Conceptual Framework (TPtoSCF).

5. TPtoSCF captures detailed information by addressing multitude 
of determinants and their related barriers in a very organized way, so that at the end of this inquiry/exercise, the gathered information may be helpful to tell us the possible social reasons linked with the death of the very child.

6. The gathered information will then be helpful to plan any intervention/s to prevent or address the possible determinant/s linked with the death, thereby improving the child survival estimates of the region in the long run.

\section{Competing Interest}

All the authors disclose that there are no competing interests in the preparation of this article.

\section{Acknowledgment}

I acknowledge all the researchers and healthcare professionals (along with all the funding agencies) who strive and give their efforts in improving women and child survival of the developing countries. Their untiring efforts should be complemented by a strong governmental support to bring a positive change.

\section{References}

1. Sadiqua N. Jafarey TR, Marge Koblinsky, Nazo Kureshy. Verbal Autopsy of Maternal Deaths in Two Districts of Pakistan-Filling Information Gaps. 2009.

2. Peter Waiswa HDK, Robert Jakob \& Robert E Black. Increased use of social autopsy is needed to improve maternal, neonatal and child health programmes in low income countries. Bulletin World Health Organization. 2012;90:403-A.

3. Xing Lin Feng T, Evropi, Li Liu, Chan, David Hipgrave, Robert Scherpbier, Kit Yee, Sufang Guo, Wen
Chunmei, Mickey Chopra, Harry Campbell, Igor Rudan, Yan Guo,. Social, economic, political and health system and program determinants of child mortality reduction in China between 1990 and 2006: A systematic analysis. Journal of global health. 2012.

4. Hinderaker SG OB, Bergsjøc PB, Gasheka P, Lied RT, Havnen J, Kvale G. Avoidable stillbirths and neonatal deaths in rural Tanzania. International Journal of Obstetrics and Gynaecology. 2003;110:616-23.

5. David Marsh NM, Zeba Rasmussen, Khalid Mateen, Arif Amin Khan. Cause-Specific Child Mortality In A Mountainous Community in Pakistan By Verbal Autopsy. JPMA. 1993;43:226-9.

6. Kallander K, Kadobera D, Williams TN, Nielsen RT, Yevoo L, Mutebi A, et al. Social autopsy: INDEPTH Network experiences of utility, process, practices, and challenges in investigating causes and contributors to mortality. Popul Health Metr. 2011;9:44.

7. Koffi AK, Maina A, Yaroh AG, Habi O, Bensaid K, Kalter HD. Social determinants of child mortality in Niger: Results from the 2012 National Verbal and Social Autopsy Study. Journal of global health. 2016;6(1):010603.

8. Shah R, Mullany LC, Darmstadt GL, Talukder RR, Rahman SM, Mannan I, et al. Determinants and pattern of care seeking for preterm newborns in a rural Bangladeshi cohort. BMC Health Serv Res. 2014;14:417.

9. Peter Waiswa KK, Stefan Peterson, Goran Tomson and George W. Pariyo. Using the three delays model to understand why newborn babies die in eastern Uganda. Tropical Medicine 
and International Health. 2010; volume 15(8):964-72.

10. M B Siddiqui CWN, Wah Yun Low. Social Autopsy is a dire need for investigating child mortality in Pakistan. International Journal of Endorsing Health Science Research [Internet]. 2016; 4(2). Available from: http://aeirc-edu.com/wpcontent/uploads/Download-full-Paper12.pdf.

11. Kalter HD, Salgado R, Babille M, Koffi AK, Black RE. Social autopsy for maternal and child deaths: A comprehensive literature review to examine the concept and the development of the method. Population Health Metrics. 2011;9.

12. Upadhyay RP, Krishnan A, Rai SK, Chinnakali P, Odukoya O. Need to focus beyond the medical causes: A systematic review of the social factors affecting neonatal deaths. Paediatric and Perinatal Epidemiology. 2014;28(2):127-37.

13. Waldman R BA, Campbell C, Steketee R:. Overcoming Remaining Barriers: The Pathway to Child Survival. Arlington, VA: United States Agency for International Development, the BASICS Project;. 1996. 